\title{
Association of Tramadol and Hypoglycemia in Diabetic Asians
}

\author{
Shang-Yi Li ${ }^{1,+}$, Hsin-Hung Chen ${ }^{2,3,4,5,+}$, Cheng-Li Lin ${ }^{6,7}$, Su-Yin Yeh ${ }^{8}$ and \\ Chia-Hung Kao $9,10,11, *$ (D) \\ 1 Department of Otorhinolaryngology, Head and Neck Surgery, Changhua Christian Hospital, Changhua 500, \\ Taiwan; 150614@cch.org.tw \\ 2 Institute of Medicine and Public Health, Chung Shan Medical University, Taichung 402, Taiwan; \\ 605319@cch.org.tw \\ 3 College of Nursing and Health Sciences, Dayeh University, Changhua 515, Taiwan \\ 4 Division of Metabolism Endocrinology, Changhua Christian Hospital, Changhua 500, Taiwan \\ Division of Metabolism Endocrinology, Nantou Christian Hospital, Nantou 540, Taiwan \\ 6 Management Office for Health Data, China Medical University Hospital, Taichung 404, Taiwan; \\ orangechengli@gmail.com \\ 7 College of Medicine, China Medical University, Taichung 404, Taiwan \\ 8 Department of Healthcare Administration, Asia University, Taichung 413, Taiwan; nch2019@yahoo.com.tw \\ 9 Graduate Institute of Biomedical Sciences and School of Medicine, College of Medicine, \\ China Medical University, No. 2, Yuh-Der Road, Taichung 404, Taiwan \\ 10 Department of Nuclear Medicine and PET Center, China Medical University Hospital, Taichung 404, Taiwan \\ 11 Department of Bioinformatics and Medical Engineering, Asia University, Taichung 404, Taiwan \\ * Correspondence: d10040@mail.cmuh.org.tw; Tel.: +886-422-052-121 (ext. 7412); Fax: +886-422-336-174 \\ $\dagger$ These authors contributed equally to the work.
}

Received: 26 September 2018; Accepted: 22 October 2018; Published: 24 October 2018

Abstract: To evaluate the association between tramadol and hypoglycemia in diabetic Asians. The data adopted in this study were derived from a subset of the National Health Insurance (NHI) Research Database, which comprises data on one million randomly sampled beneficiaries enrolled in the NHI program. Patients diagnosed with diabetes (according to the International Classification of Diseases, Ninth Revision, Clinical Modification code 250) were identified from claims data between 1998 and 2011. Diabetic patients aged 20 years or older and prescribed tramadol constituted the tramadol group and other diabetic patients without tramadol use constituted the non-tramadol group. For each tramadol case, one non-tramadol control frequency matched according to age (every 5 years), sex and the year of tramadol use was identified. The tramadol group comprised 12,446 patients and non-tramadol group comprised 11,982 patients. During a mean follow-up of 2 years for the patients in the tramadol group and 2.79 years for those in the non-tramadol group, the overall incidences of hypoglycemia (per 1000 person-years) were 7.37 and 3.77, respectively. According to the multivariable analyses, after baseline characteristics were controlled, the tramadol group exhibited a significantly greater risk of hypoglycemia (hazard ratio $(\mathrm{HR})=1.34,95 \%$ confidence interval $(\mathrm{CI})=1.05-1.71$ ) compared with the non-tramadol group. Tramadol use increases hypoglycemia in diabetic Asians. Greater attention must be paid to diabetic Asians with tramadol use.

Keywords: tramadol; hypoglycemia; diabetes

\section{Introduction}

Hypoglycemia poses a challenge in managing diabetes. The action to control cardiovascular risk in diabetes study showed that hypoglycemia caused considerable mortalities [1]. In addition, the action in 
diabetes and vascular disease: Preterax and diamicron modified release controlled evaluation indicated that hypoglycemia increases microvascular as well as macrovascular comorbidities and mortalities [2]. Clinical symptomatic hypoglycemia causes cardiovascular events, all-cause hospitalization and mortality [3]. The risk of dementia increased in older diabetic patients [4]. Cognitive impairment was significantly associated with severe hypoglycemia [5]. In 2013, the American Diabetes Association and the Endocrine Society published a consensus report called "Hypoglycemia and Diabetes [6]." Thus, how to avoid hypoglycemic attacks in diabetic patients has been extensively discussed worldwide.

According to Real-life Effectiveness and Care Patterns of Diabetes Management [7], 29.4\% of diabetic patients suffered a hypoglycemic attack. Tramadol is one of the medications prescribed by the American Diabetes Association for treating painful diabetic peripheral neuropathy [8]. However, tramadol therapy with non-cancer pain could increase the risk of hypoglycemia and lead to hospitalization [9]. A previous study indicated that hypoglycemia is one of the major reasons for emergency hospitalizations for adverse drug events in older Americans [10], reporting that insulin, instead of tramadol, caused emergency hospitalizations in the United States of America. The objective of the present study was to evaluate the association between tramadol and hypoglycemia in diabetic Asians.

\section{Methods}

\subsection{Data Source}

The National Health Insurance (NHI) program in Taiwan was implemented in 1995 and provides comprehensive medical care, including ambulatory and inpatient care, to nearly $100 \%$ of Taiwan's population, which is approximately 23.72 million people [11]. The National Health Insurance Research Database (NHIRD) contains historical reimbursement claims data from the NHI program and is maintained and managed by the National Health Research Institute. The data used in this study were derived from a subset of the NHIRD, which comprises data on one million randomly sampled beneficiaries enrolled in the NHI program from 1996 to 2000. All records of these individuals from 1996 to 2011 were collected. The data files were de-identified and scrambled before being released to researchers. Therefore, this database cannot be used to query the data alone to identify individuals at any level. The diagnoses and procedures were coded according to the International Classification of Diseases, Ninth Revision, Clinical Modification (ICD-9-CM).

\subsection{Sampled Participants}

Patients diagnosed with diabetes (ICD-9-CM code 250) were identified from the claims data between 1998 and 2011. Diabetic patients aged 20 years or older and prescribed tramadol constituted the tramadol group and those who did not use tramadol constituted the non-tramadol group. The initial tramadol treatment date was defined as the index date. For each tramadol case, one non-tramadol control frequency matched according to age (every 5 years), sex and the year of tramadol use was identified. Controls were randomly assigned the same index date as the tramadol group. Patients in both groups with a history of hypoglycemia (ICD-9-CM codes 251.0, 251.1 and 251.2) or with incomplete information were excluded.

\subsection{Outcome}

Both the tramadol and non-tramadol groups were followed from the index date to the date of hypoglycemia diagnosis, withdrawal from the NHI program, censoring because of death, or end date of the database (31 December 2011).

\subsection{Comorbidities and Medications}

The records of comorbidities and medications were obtained before the index date. Comorbidities included acute myocardial infarction (ICD-9-CM code 410), chronic kidney disease (ICD-9-CM 
codes 580-589), stroke (ICD-9-CM codes 430-438), hypertension (ICD-9-CM codes 401-405), cancer (ICD-9-CM codes 140-208) and alcohol-related illness (ICD-9-CM codes 291, 303, 305, 571.0, 571.1, 571.2, 571.3, 790.3, A215 and V11.3). Medications included antidiabetic drugs (including alpha-glucosidase inhibitors, sulfonylurea, metformin, thiazolidinedione, insulin and dipeptidyl peptidase 4), statins, aspirin, nonsteroidal anti-inflammatory drugs, antihypertensive agents (including angiotensin-converting enzyme inhibitors, angiotensin II receptor blockers (AIIRBs), calcium channel blockers (dihydropyridine) (CCB (DHP)), $\alpha$-blockers, $\beta$-blockers, CCB (non-DHP), loop diuretics and thiazides), antiarrhythmics, serotonin reuptake inhibitors, benzodiazepine, tricyclic antidepressants (TCA), serotonin-norepinephrine reuptake inhibitors (SNRI) and other opioid.

\subsection{Statistical Analysis}

The distributions of age, sex, comorbidities and medications between the groups with and without tramadol use were compared using chi-square tests for categorical variables and $t$ tests for continuous variables. The incidence densities (per 1000 person-years) of hypoglycemia were estimated in both the tramadol and non-tramadol groups. Univariable and multivariable Cox proportional hazards regression models were employed to estimate hazard ratios (HRs) and 95\% confidence intervals (CIs) for hypoglycemia in in patients in the tramadol group compared with those in the non-tramadol group. Baseline characteristic variables such as sex, age, comorbidities and medications were included in the multivariate model for adjustment. In the multivariable model, antidiabetic drugs, including sulfonylurea, metformin and insulin and antihypertensive agents, including loop diuretics, attained significance. Additional data analysis was performed to evaluate the interaction among tramadol use, sulfonylurea, metformin, insulin and loop diuretics. The cumulative incidences of hypoglycemia between the tramadol and non-tramadol groups were assessed using the Kaplan-Meier method and the differences were assessed using a log-rank test. All statistical analyses were performed using the SAS statistical package (Version 9.3 for Windows; SAS Institute, Inc., Cary, NC, USA). Statistical significance was accepted at $p<0.05$.

\subsection{Data Availability Statement}

All data and related metadata were deposited in an appropriate public repository. The data on the study population that were obtained from the NHIRD (http:/ / w3.nhri.org.tw / nhird/ / date_01.html) are maintained in the NHIRD (http:/ / nhird.nhri.org.tw/). The National Health Research Institutes (NHRI) is a nonprofit foundation established by the government.

\subsection{Ethics Statement}

The NHIRD encrypts patient personal information to protect privacy and provides researchers with anonymous identification numbers associated with relevant claims information, including sex, date of birth, medical services received and prescriptions. Patient consent is not required to access the NHIRD. This study was approved by the Institutional Review Board (IRB) of China Medical University (CMUH104-REC2-115-CR3). The IRB specifically waived the consent requirement.

\section{Results}

In this study, the tramadol group comprised 12,446 patients and non-tramadol group comprised 11,982 patients (Table 1). Among the patients in the tramadol group, 56.9\% were older than 65 years and $50.8 \%$ were women. The mean ages of the patients in the tramadol and non-tramadol groups were 66.3 (standard deviation $(\mathrm{SD})=12.6)$ and $65.4(\mathrm{SD}=12.2)$ years, respectively. Diabetic patients in the tramadol group were more likely to have acute myocardial infarction, chronic kidney disease, stroke, hypertension, cancer or alcohol-related diseases $(p<0.001)$ compared with those in the non-tramadol group. At the baseline, all the medications were more prevalent in the tramadol group $(p<0.001)$ compared with the non-tramadol group. 
Table 1. Comparison of baseline characteristics between diabetes patients with and without tramadol use.

\begin{tabular}{|c|c|c|c|c|c|}
\hline \multirow{4}{*}{ Variables } & \multicolumn{4}{|c|}{ Tramadol } & \multirow{4}{*}{$p$ Value } \\
\hline & \multirow{2}{*}{\multicolumn{2}{|c|}{$\begin{array}{c}\text { No } \\
n=11,982\end{array}$}} & \multirow{2}{*}{\multicolumn{2}{|c|}{$\begin{array}{c}\text { Yes } \\
n=12,446\end{array}$}} & \\
\hline & & & & & \\
\hline & $n$ & $(\%)$ & $n$ & $(\%)$ & \\
\hline Age, year & & & & & 0.05 \\
\hline$\leq 34$ & 1311 & 10.9 & 1311 & 10.5 & \\
\hline $35-64$ & 4058 & 33.9 & 4058 & 32.6 & \\
\hline $65+$ & 6613 & 55.2 & 7077 & 56.9 & \\
\hline Mean (SD) & 65.4 & 12.2 & 66.3 & 12.6 & $<0.001^{\dagger}$ \\
\hline Sex & & & & & 0.99 \\
\hline Female & 6085 & 50.8 & 6322 & 50.8 & \\
\hline Male & 5897 & 49.2 & 6124 & 49.2 & \\
\hline Comorbidity & & & & & \\
\hline Acute myocardial infarction & 227 & 1.89 & 354 & 2.84 & $<0.001$ \\
\hline Chronic kidney disease & 1931 & 16.1 & 3380 & 27.2 & $<0.001$ \\
\hline Stroke & 1712 & 14.3 & 2120 & 17.0 & $<0.001$ \\
\hline Hypertension & 9343 & 78.0 & 9932 & 79.8 & $<0.001$ \\
\hline Cancer & 618 & 5.16 & 2120 & 17.0 & $<0.001$ \\
\hline Alcohol-related diseases & 629 & 5.25 & 1267 & 10.2 & $<0.001$ \\
\hline Medication & & & & & \\
\hline Alpha-glucosidase inhibitors & 1899 & 15.9 & 3360 & 27.0 & $<0.001$ \\
\hline Sulfonylurea & 7329 & 61.2 & 9241 & 74.3 & $<0.001$ \\
\hline Metformin & 7935 & 66.2 & 9571 & 76.9 & $<0.001$ \\
\hline Thiazolidinediones & 1534 & 12.8 & 2998 & 24.1 & $<0.001$ \\
\hline Others & 83 & 0.69 & 227 & 1.82 & $<0.001$ \\
\hline Insulin & 6416 & 53.6 & 9244 & 74.3 & $<0.001$ \\
\hline Dpp4 & 411 & 3.43 & 819 & 6.58 & $<0.001$ \\
\hline Statin & 5272 & 44.0 & 6259 & 50.3 & $<0.001$ \\
\hline Aspirin & 7102 & 59.3 & 8379 & 67.3 & $<0.001$ \\
\hline NSAID & 11,574 & 96.6 & 12,316 & 99.0 & $<0.001$ \\
\hline ACEI & 7086 & 59.1 & 8278 & 66.5 & $<0.001$ \\
\hline AIIRB & 5301 & 44.2 & 6516 & 52.4 & $<0.001$ \\
\hline CCB (DHP) & 8129 & 67.8 & 9316 & 74.9 & $<0.001$ \\
\hline$\alpha$-Blockers & 3490 & 29.1 & 4330 & 34.8 & $<0.001$ \\
\hline$\beta$-Blockers & 7834 & 65.4 & 9096 & 73.1 & $<0.001$ \\
\hline CCB (non-DHP) & 3927 & 32.8 & 5055 & 40.6 & $<0.001$ \\
\hline Loop diuretics & 4769 & 39.8 & 7695 & 61.8 & $<0.001$ \\
\hline Thiazides & 6914 & 57.7 & 7986 & 64.2 & $<0.001$ \\
\hline Antiarrhythmics & 461 & 3.85 & 688 & 5.53 & $<0.001$ \\
\hline Serotonin reuptake inhibitors & 334 & 2.79 & 556 & 4.47 & $<0.001$ \\
\hline Benzodiazepine & 10,308 & 86.0 & 11,768 & 94.6 & $<0.001$ \\
\hline TCA & 617 & 5.15 & 1285 & 10.3 & $<0.001$ \\
\hline SNRI & 133 & 1.11 & 330 & 2.65 & $<0.001$ \\
\hline Other opioid & 8627 & 72.0 & 10,916 & 87.7 & $<0.001$ \\
\hline Year & & & & & \\
\hline 1998 & & & 1555 & 12.5 & \\
\hline 1999 & & & 2197 & 17.7 & \\
\hline 2000 & & & 1886 & 15.2 & \\
\hline 2001 & & & 977 & 7.85 & \\
\hline 2002 & & & 864 & 6.94 & \\
\hline 2003 & & & 734 & 5.90 & \\
\hline 2004 & & & 845 & 6.79 & \\
\hline 2005 & & & 710 & 5.70 & \\
\hline
\end{tabular}


Table 1. Cont.

\begin{tabular}{|c|c|c|c|c|c|}
\hline \multirow{4}{*}{ Variables } & \multicolumn{4}{|c|}{ Tramadol } & \multirow{4}{*}{$p$ Value } \\
\hline & \multirow{2}{*}{\multicolumn{2}{|c|}{$\begin{array}{c}\text { No } \\
n=11,982 \\
\end{array}$}} & \multirow{2}{*}{\multicolumn{2}{|c|}{$\begin{array}{c}\text { Yes } \\
n=12,446\end{array}$}} & \\
\hline & & & & & \\
\hline & $n$ & $(\%)$ & $n$ & $(\%)$ & \\
\hline \multicolumn{6}{|l|}{ Year } \\
\hline 2006 & & & 616 & 4.95 & \\
\hline 2007 & & & 618 & 4.97 & \\
\hline 2008 & & & 532 & 4.27 & \\
\hline 2009 & & & 319 & 2.56 & \\
\hline 2010 & & & 118 & 0.95 & \\
\hline
\end{tabular}

Chi-square test; ${ }^{\dagger} t$-test; AIIRB, angiotensin II receptor blocker; CCB (DHP), calcium channel blockers (dihydropyridine); SD, standard deviation; TCA, tricyclic antidepressants; SNRI, serotonin-norepinephrine reuptake inhibitors; Dpp4, dipeptidyl peptidase-4 inhibitors; NSAID, non-steroidal anti-inflammatory drug; ACEI, angiotension-converting enzyme inhibitors.

During the mean follow up of 2 years for the tramadol group and 2.79 years for the non-tramadol group, the overall incidences of hypoglycemia (per 1000 person-years) were 7.37 and 3.77, respectively (Table 2). According to the multivariable analyses, after baseline characteristics were controlled, the patients in the tramadol group exhibited a significantly greater risk of hypoglycemia $(\mathrm{HR}=1.32$, $95 \% \mathrm{CI}=1.03-1.68)$ compared with those in the non-tramadol group. The incidence of hypoglycemia increased with age in both groups. The relative risk of hypoglycemia in the sex-specific tramadol group to non-tramadol group was significant for men ( $\mathrm{HR}=1.66,95 \% \mathrm{CI}=1.10-2.50)$. We analyzed the association between tramadol use and the risk of hypoglycemia stratified according to cancer and observed an approximate 1.33-fold hypoglycemia risk in patients with tramadol use and without cancer $(\mathrm{HR}=1.33,95 \% \mathrm{CI}=1.03-1.71)$. The cumulative incidence of hypoglycemia $(\log$-rank $p<0.001)$ was significantly higher for patients with tramadol use and without cancer than it was for patients without tramadol use and without cancer (Figure 1). The sex-specific hazard of hypoglycemia in patients with tramadol use and without cancer relative to that in patients without tramadol use and without cancer was significant for men $(\mathrm{HR}=1.60,95 \% \mathrm{CI}=1.05-2.43)$.

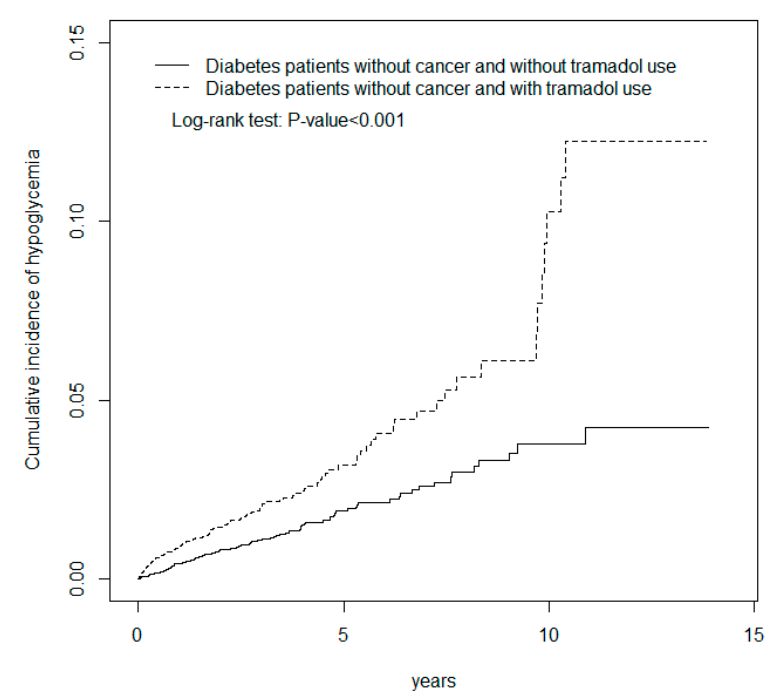

Figure 1. Cumulative incidence of hypoglycemia for patients with (dashed line) or without (solid line) tramadol use among diabetes patients without cancer. 
Table 2. Comparisons of hypoglycemia incidences between diabetes patients with and without tramadol use and associated hazard ratios by age, sex and comorbidity.

\begin{tabular}{|c|c|c|c|c|c|c|c|c|}
\hline \multirow{3}{*}{ Variables } & \multicolumn{6}{|c|}{ Tramadol } & \multirow{3}{*}{ Crude HR (95\% CI) } & \multirow{3}{*}{ Adjusted $\mathrm{HR}^{\dagger}(95 \% \mathrm{CI})$} \\
\hline & \multicolumn{3}{|c|}{$\begin{array}{l}\text { No } \\
\end{array}$} & \multicolumn{3}{|c|}{ Yes } & & \\
\hline & Event & PY & Rate $^{\#}$ & Event & PY & Rate $^{\#}$ & & \\
\hline All & 126 & 33,465 & 3.77 & 183 & 24,838 & 7.37 & $1.94(1.55,2.44)^{* * *}$ & $1.32(1.03,1.68)$ * \\
\hline \multicolumn{9}{|l|}{ Age, years } \\
\hline$\leq 49$ & 2 & 4021 & 0.05 & 11 & 3162 & 3.48 & $7.70(1.70,34.9)^{* *}$ & $4.10(0.70,24.2)$ \\
\hline $50-64$ & 20 & 11,696 & 1.71 & 38 & 8432 & 4.51 & $2.66(1.54,4.60)^{* * *}$ & $1.62(0.90,2.92)$ \\
\hline $65+$ & 104 & 17,748 & 5.86 & 134 & 13,244 & 10.1 & $1.71(1.32,2.21)^{* * *}$ & $1.26(0.95,1.66)$ \\
\hline \multicolumn{9}{|l|}{ Sex } \\
\hline Female & 83 & 16,658 & 4.98 & 107 & 13,292 & 8.05 & $1.61(1.21,2.15)^{* *}$ & $1.17(0.86,1.60)$ \\
\hline Male & 43 & 16,807 & 2.56 & 76 & 11,546 & 6.58 & $2.56(1.75,3.74)^{* * *}$ & $1.66(1.10,2.50) *$ \\
\hline \multicolumn{9}{|l|}{ With cancer } \\
\hline All & 4 & 1324 & 3.02 & 18 & 2905 & 6.19 & $1.88(0.63,5.57)$ & $1.53(0.49,4.78)$ \\
\hline \multicolumn{9}{|l|}{ Age, years } \\
\hline$\leq 49$ & 0 & 43 & 0.00 & 1 & 304 & 3.29 & - & - \\
\hline $50-64$ & 0 & 378 & 0.00 & 8 & 1014 & 7.89 & - & - \\
\hline $65+$ & 4 & 904 & 4.43 & 9 & 1588 & 5.67 & $1.24(0.38,4.07)$ & $1.26(0.33,4.79)$ \\
\hline \multicolumn{9}{|l|}{ Sex } \\
\hline Female & 1 & 744 & 4.03 & 10 & 1352 & 7.39 & $1.72(0.47,6.30)$ & $2.05(0.44,9.54)$ \\
\hline Male & 3 & 581 & 1.72 & 8 & 1553 & 5.15 & $2.60(0.32,20.9)$ & $1.92(0.18,20.2)$ \\
\hline \multicolumn{9}{|l|}{$\begin{array}{l}\text { Without } \\
\text { cancer }\end{array}$} \\
\hline All & 122 & 32,140 & 3.80 & 165 & 21,932 & 7.52 & $1.97(1.56,2.50)^{* * *}$ & $1.33(1.03,1.71)$ * \\
\hline \multicolumn{9}{|l|}{ Age, years } \\
\hline$\leq 49$ & 2 & 3978 & 0.50 & 10 & 2858 & 3.50 & $7.89(1.72,36.2)^{* *}$ & $4.61(0.75,28.3)$ \\
\hline $50-64$ & 100 & 11,318 & 1.77 & 30 & 7417 & 4.04 & $2.31(1.30,4.09) * *$ & $1.39(0.75,2.58)$ \\
\hline $65+$ & 10 & 16,844 & 5.94 & 125 & 11,656 & 10.7 & $1.79(1.37,2.34)^{* * *}$ & $1.30(0.98,1.73)$ \\
\hline \multicolumn{9}{|l|}{ Sex } \\
\hline Female & 80 & 15,914 & 5.03 & 97 & 11,939 & 8.12 & $1.62(1.20,2.18)^{* *}$ & $1.20(0.87,1.65)$ \\
\hline Male & 42 & 16,226 & 2.59 & 68 & 9992 & 6.80 & $2.60(1.77,3.84)^{* * *}$ & $1.60(1.05,2.43)$ * \\
\hline
\end{tabular}

Rate \#, incidence rate, per 1000 person-years; Crude HR, relative hazard ratio; Adjusted $\mathrm{HR}^{\dagger}{ }^{\dagger}$, multivariable analysis including age, sex, comorbidities of acute myocardial infarction, chronic kidney disease, stroke, hypertension and medications of alpha-glucosidase inhibitors, sulfonylurea, metformin, thiazolidinediones, insulin, others, aspirin, ACEI, angiotensin II receptor blocker (AIIRB), calcium channel blockers (dihydropyridine) (CCB (DHP)), $\alpha$-Blockers, $\beta$-Blockers, CCB (non-DHP), loop diuretics, thiazides, antiarrhythmics, serotonin reuptake inhibitors, benzodiazepine and TCA; ${ }^{*} p<0.05,{ }^{* *} p<0.01,{ }^{* * *} p<0.001$; CI, confidence interval; HR, hazard ratio; PY, person-years; TCA, tricyclic antidepressants; ACEI, angiotension-converting enzyme inhibitors.

Table 3 shows the results of the univariable and multivariable Cox proportional hazard analyses conducted for evaluating the association between hypoglycemia and tramadol use. The adjusted HR of hypoglycemia development was a 1.47-fold increase for men compared with that of women $(\mathrm{HR}=1.36$, $95 \% \mathrm{CI}=1.05-1.76)$ and a 1.05 -fold increase $(95 \% \mathrm{CI}=1.04-1.06)$ with age (every 1 year). The risk of hypoglycemia was higher for patients with chronic kidney disease ( $\mathrm{HR}=1.36,95 \% \mathrm{CI}=1.06-1.75)$ and medications of sulfonylurea $(\mathrm{HR}=1.72,95 \% \mathrm{CI}=1.18-2.51)$, metformin $(\mathrm{HR}=1.74,95 \% \mathrm{CI}=1.20-2.53)$, insulin ( $\mathrm{HR}=1.86,95 \% \mathrm{CI}=1.32-2.61)$ and loop diuretics $(\mathrm{HR}=1.32,95 \% \mathrm{CI}=1.01-1.73)$. Table 4 shows the joint effect of tramadol, sulfonylurea, metformin, insulin, loop diuretics, benzodiazepine, TCA, SNRI and other opioid use on the risk of hypoglycemia. Compared with the patients without tramadol and sulfonylurea use, the patients with tramadol and sulfonylurea use exhibited an adjusted HR of $2.64(95 \% \mathrm{CI}=1.56-4.44)$. The HR increased to $2.24(95 \% \mathrm{CI}=1.49-3.36)$ for the patients with tramadol and insulin use. The risk of hypoglycemia for patients in the tramadol group was increased with metformin ( $\mathrm{HR}=2.23,95 \% \mathrm{CI}=1.39-3.59)$ and loop diuretics use $(\mathrm{HR}=1.71,95 \% \mathrm{CI}=1.21-2.43)$. Compared with the patients without tramadol and TCA use, the patients with tramadol and TCA use exhibited an adjusted HR of 1.61 (95\% CI $=1.05-2.44)$. 
Table 3. Cox model with hazard ratios and $95 \%$ confidence intervals of hypoglycemia associated with tramadol use and covariates among diabetes patients without cancer.

\begin{tabular}{|c|c|c|c|c|}
\hline \multirow{2}{*}{ Variable } & \multicolumn{2}{|c|}{ Crude } & \multicolumn{2}{|c|}{ Adjusted $^{+}$} \\
\hline & HR & $(95 \% \mathrm{CI})$ & HR & $(95 \% \mathrm{CI})$ \\
\hline Gender (women vs. men) & 1.52 & $(1.19,1.92)^{* * *}$ & 1.36 & $(1.05,1.76) *$ \\
\hline Age, years & 1.05 & $(1.04,1.06)^{* * *}$ & 1.05 & $(1.04,1.06) *$ \\
\hline \multicolumn{5}{|l|}{ Baseline comorbidities (yes vs. no) } \\
\hline Acute myocardial infarction & 1.92 & $(1.08,3.43) *$ & 1.18 & $(0.66,2.11)$ \\
\hline Chronic kidney disease & 1.99 & $(1.56,2.54)^{* * *}$ & 1.36 & $(1.06,1.75)$ * \\
\hline Stroke & 1.67 & $(1.25,2.22)^{* * *}$ & & \\
\hline Hypertension & 2.07 & $(1.46,2.95)^{* * *}$ & 0.83 & $(0.53,1.30)$ \\
\hline Cancer & 0.94 & $(0.61,1.46)$ & 0.75 & $(0.48,1.16)$ \\
\hline Alcohol-related diseases & 0.93 & $(0.58,1.49)$ & & \\
\hline \multicolumn{5}{|l|}{ Medication (yes vs. no) } \\
\hline Tramadol & 1.97 & $(1.56,2.50)^{* * *}$ & 1.32 & $(1.03,1.68)$ * \\
\hline Alpha-glucosidase inhibitors & 2.01 & $(1.55,2.60)^{* * *}$ & 1.20 & $(0.90,1.58)$ \\
\hline Sulfonylurea & 3.09 & $(2.19,4.34)^{* * *}$ & 1.72 & $(1.18,2.51)^{* *}$ \\
\hline Metformin & 2.71 & $(1.95,3.77)^{* * *}$ & 1.74 & $(1.20,2.53)^{* *}$ \\
\hline Thiazolidinediones & 1.64 & $(1.24,2.16)^{* * *}$ & 0.97 & $(0.73,1.31)$ \\
\hline Others & 2.41 & $(1.19,4.86) *$ & 1.53 & $(0.78,3.01)$ \\
\hline Insulin & 3.28 & $(2.42,4.45)^{* * *}$ & 1.86 & $(1.32,2.61)^{* *}$ \\
\hline Dpp4 & 0.94 & $(0.42,2.13)$ & & \\
\hline Statin & 1.07 & $(0.85,1.36)$ & & \\
\hline Aspirin & 1.46 & $(1.14,1.87)^{* *}$ & 0.82 & $(0.62,1.08)$ \\
\hline NSAID & 1.83 & $(0.81,4.14)$ & & \\
\hline ACEI & 2.18 & $(1.66,2.87)^{* * *}$ & 1.23 & $(0.89,1.70)$ \\
\hline AIIRB & 1.32 & $(1.04,1.68) *$ & 0.72 & $(0.55,0.94) *$ \\
\hline CCB (DHP) & 2.18 & $(1.62,2.94)^{* * *}$ & 1.38 & $(0.96,1.99)$ \\
\hline$\alpha$-Blockers & 1.69 & $(1.34,2.14)^{* * *}$ & 1.24 & $(0.95,1.62)$ \\
\hline$\beta$-Blockers & 1.64 & $(1.25,2.14)^{* * *}$ & 1.05 & $(0.78,1.42)$ \\
\hline CCB (non-DHP) & 1.64 & $(1.30,2.07)^{* * *}$ & 1.05 & $(0.82,1.35)$ \\
\hline Loop diuretics & 2.38 & $(1.86,3.03)^{* * *}$ & 1.32 & $(1.01,1.73)$ * \\
\hline Thiazides & 1.85 & $(1.43,2.39)^{* * *}$ & 1.03 & $(0.77,1.38)$ \\
\hline Antiarrhythmics & 1.87 & $(1.19,2.95)^{* *}$ & 1.22 & $(0.77,1.95)$ \\
\hline Serotonin reuptake inhibitors & 1.64 & $(1.00,2.67) *$ & 1.33 & $(0.81,2.18)$ \\
\hline Benzodiazepine & 1.92 & $(1.23,2.99)^{* *}$ & 0.97 & $(0.60,1.55)$ \\
\hline TCA & 1.47 & $(1.03,2.11) *$ & 1.05 & $(0.73,1.52)$ \\
\hline SNRI & 1.42 & $(0.67,3.00)$ & & \\
\hline Other opioid & 1.26 & $(0.95,1.66)$ & & \\
\hline
\end{tabular}

Crude $\mathrm{HR}$, relative hazard ratio; Adjusted $\mathrm{HR}^{\dagger}{ }^{\dagger}$, multivariable analysis including age, sex and comorbidities of acute myocardial infarction, chronic kidney disease, stroke, hypertension and medications of alpha-glucosidase inhibitors, sulfonylurea, metformin, thiazolidinediones, insulin, others, aspirin, ACEI, AIIRB, calcium channel blockers (dihydropyridine) (CCB (DHP)), $\alpha$-Blockers, $\beta$-Blockers, CCB (non-DHP), loop diuretics, thiazides, antiarrhythmics, serotonin reuptake inhibitors, benzodiazepine and TCA; $*<0.05, * * p<0.01, * * *<0.001$; CI, confidence interval; HR, hazard ratio; AIIRB, angiotensin II receptor blockers; TCA, tricyclic antidepressants; SNRI, serotonin-norepinephrine reuptake inhibitors; Dpp4, dipeptidyl peptidase-4 inhibitors; NSAID, non-steroidal anti-inflammatory drug; ACEI, angiotension-converting enzyme inhibitors.

Table 4. Cox proportional hazard regression analysis for the risk of hypoglycemia-associated tramadol use with joint effect of medication.

\begin{tabular}{ccccccc}
\hline \multicolumn{2}{c}{ Variables } & Event & PY & Rate $^{\&}$ & Adjusted HR $^{\dagger} \mathbf{( 9 5 \% ~ C I )}$ & $p_{\text {-Value }}{ }^{\#}$ \\
\hline Tramadol use & Sulfonylurea & & & & & \\
No & No & 19 & 12,188 & 1.56 & 1 (Reference) & \\
No & Yes & 107 & 21,276 & 5.03 & $2.11(1.26,3.54)^{*}$ & 0.55 \\
Yes & No & 21 & 6315 & 3.33 & $1.91(1.02,3.57)^{* * *}$ & \\
Yes & Yes & 162 & 18,522 & 8.75 & $2.64(1.56,4.44)^{*}$ & \\
\hline
\end{tabular}


Table 4. Cont.

\begin{tabular}{|c|c|c|c|c|c|c|}
\hline \multicolumn{2}{|c|}{ Variables } & \multirow[t]{2}{*}{ Event } & \multirow[t]{2}{*}{ PY } & \multirow[t]{2}{*}{ Rate $^{\&}$} & \multirow[t]{2}{*}{ Adjusted $\mathrm{HR}^{\dagger}(95 \% \mathrm{CI})$} & \multirow[t]{2}{*}{$p$-Value ${ }^{\#}$} \\
\hline Tramadol use & Metformin & & & & & \\
\hline No & No & 25 & 11,954 & 2.09 & 1 (Reference) & \\
\hline No & Yes & 101 & 21,510 & 4.70 & $1.65(1.03,2.64)$ * & 0.29 \\
\hline Yes & No & 17 & 6169 & 2.76 & $1.15(0.62,2.15)$ & \\
\hline Yes & Yes & 166 & 18,669 & 8.89 & $2.23(1.39,3.59) * * *$ & \\
\hline Tramadol use & Insulin & & & & & \\
\hline No & No & 36 & 16,590 & 2.17 & 1 (Reference) & \\
\hline No & Yes & 90 & 16,876 & 5.33 & $1.57(1.05,2.37) *$ & 0.07 \\
\hline Yes & No & 15 & 7087 & 2.12 & $0.91(0.50,1.68)$ & \\
\hline Yes & Yes & 168 & 17,751 & 9.46 & $2.24(1.49,3.36)^{* * *}$ & \\
\hline Tramadol use & Loop diuretics & & & & & \\
\hline No & No & 62 & 21,994 & 2.82 & 1 (Reference) & \\
\hline No & Yes & 64 & 11,471 & 5.58 & $1.24(0.86,1.81)$ & 0.78 \\
\hline Yes & No & 46 & 10,451 & 4.40 & $1.24(0.83,1.83)$ & \\
\hline Yes & Yes & 137 & 14,386 & 9.52 & $1.71(1.21,2.43)^{* *}$ & \\
\hline Tramadol use & Benzodiazepine & & & & & \\
\hline No & No & 16 & 5659 & 2.83 & 1 (Reference) & \\
\hline No & Yes & 110 & 27,806 & 3.96 & $0.89(0.52,1.55)$ & 0.39 \\
\hline Yes & No & 5 & 1457 & 3.43 & $1.00(0.37,2.76)$ & \\
\hline Yes & Yes & 178 & 23,381 & 7.61 & $1.22(0.70,2.13)$ & \\
\hline Tramadol use & TCA & & & & & \\
\hline No & No & 123 & 31,876 & 3.86 & 1 (Reference) & \\
\hline No & Yes & 3 & 1589 & 1.89 & $0.37(0.12,1.18)$ & 0.06 \\
\hline Yes & No & 153 & 22,032 & 6.94 & $1.24(0.96,1.60)$ & \\
\hline Yes & Yes & 30 & 2806 & 10.7 & $1.61(1.05,2.44) *$ & \\
\hline Tramadol use & SNRI & & & & & \\
\hline No & No & 124 & 33,196 & 3.74 & 1 (Reference) & \\
\hline No & Yes & 2 & 270 & 7.42 & $1.73(0.42,7.10)$ & 0.43 \\
\hline Yes & No & 178 & 24,166 & 7.37 & $1.36(1.06,1.74)$ * & \\
\hline Yes & Yes & 5 & 672 & 7.44 & $1.24(0.49,3.10)$ & \\
\hline Tramadol use & Other opioid & & & & & \\
\hline No & No & 42 & 10,935 & 3.84 & 1 (Reference) & \\
\hline No & Yes & 84 & 22,530 & 3.73 & $0.82(0.56,1.21)$ & 0.05 \\
\hline Yes & No & 21 & 3240 & 6.48 & $1.19(0.70,2.03)$ & \\
\hline Yes & Yes & 162 & 21,598 & 7.50 & $1.16(0.79,1.69)$ & \\
\hline Tramadol use & NSAID & & & & & \\
\hline No & No & 4 & 1808 & 2.21 & 1 (Reference) & \\
\hline No & Yes & 122 & 31,657 & 3.85 & $1.43(0.52,3.94)$ & 0.25 \\
\hline Yes & No & 3 & 287 & 10.4 & $4.39(0.98,19.8)$ & \\
\hline Yes & Yes & 180 & 24,551 & 7.33 & $1.88(0.68,5.20)$ & \\
\hline
\end{tabular}

Rate $^{\&}$, incidence rate, per 1000 person-years; ${ }^{\dagger}$ Model was mutually adjusted for age, sex, comorbidities of acute myocardial infarction, chronic kidney disease, stroke, hypertension and medications of alpha-glucosidase inhibitors, sulfonylurea, metformin, thiazolidinediones, insulin, others, aspirin, ACEI, AIIRB, calcium channel blockers (dihydropyridine) (CCB (DHP)), $\alpha$-Blockers, $\beta$-Blockers, CCB (non-DHP), loop diuretics, thiazides, antiarrhythmics, serotonin reuptake inhibitors, benzodiazepine and TCA. ${ }^{*} p$-value for interaction, ${ }^{*} p<0.05,{ }^{* *} p<0.01,{ }^{* * *} p<0.001$; CI, confidence interval; HR, hazard ratio; AIIRB, angiotensin II receptor blockers; PY, person-years; TCA, tricyclic antidepressants; NSAID, non-steroidal anti-inflammatory drug; ACEI, angiotension-converting enzyme inhibitors; SNRI, serotonin-norepinephrine reuptake inhibitors.

\section{Discussion}

\subsection{Tramadol and Hypoglycemia in Diabetes}

Tramadol is one of the medications prescribed by the American Diabetes Association for treating painful diabetic peripheral neuropathy [8]. Table 1 shows that among the patients in the tramadol group, $56.9 \%$ were older than 65 years. The mean ages of the patients in the tramadol and non-tramadol groups were $66.3(\mathrm{SD}=12.6)$ and $65.4(\mathrm{SD}=12.2)$ years, respectively. The diabetic patients in the tramadol group were more likely to have acute myocardial infarction, chronic kidney disease, stroke, hypertension, or cancer $(p<0.001)$ compared with those in the non-tramadol group. At the baseline, 
all the medications were more prevalent in the tramadol group $(p<0.001)$ compared with the non-tramadol group. This finding is consistent with that of previous studies $[3,9,10,12]$. Table 2 shows that the overall incidences of hypoglycemia (per 1000 person-years) in the tramadol and non-tramadol groups were 7.37 and 3.77, respectively. The results of the multivariable analyses indicated that the patients in the tramadol group exhibited a significantly higher risk of hypoglycemia ( $\mathrm{HR}=1.34,95 \% \mathrm{CI}=1.05-1.71)$ compared with those in the non-tramadol group. Some possible mechanisms could explain that tramadol induced hypoglycemia. A previous study conducted on mice and rats indicated that serotonin increased insulin concentration and induced $\beta$-endorphin release to stimulate muscle glucose utilization. The pathway activates $\mu$-opioid receptors $[13,14]$. Another study indicated that $\mu$-opioid receptor activation reduced plasma glucose concentrations through an insulin-independent mechanism [15]. These possible mechanisms explained our finding (Table 2 and Figure 1), which indicates an approximate 1.33-fold hypoglycemia risk in the patients with tramadol use and without cancer $(\mathrm{HR}=1.33,95 \% \mathrm{CI}=1.03-1.71)$ and a significantly higher cumulative incidence of hypoglycemia (log-rank $p<0.001)$ in the patients with tramadol use and without cancer.

\subsection{Tramadol Combined with Other Medications in Diabetes}

As shown in Table 3, the risk of hypoglycemia was higher for the diabetic patients with the medications of sulfonylurea ( $\mathrm{HR}=1.76,95 \% \mathrm{CI}=1.19-2.60)$, metformin $(\mathrm{HR}=1.66,95 \% \mathrm{CI}=1.14-2.43)$, insulin ( $\mathrm{HR}=1.78,95 \% \mathrm{CI}=1.26-2.52)$ and loop diuretics ( $\mathrm{HR}=1.33,95 \% \mathrm{CI}=1.01-1.76)$ but lower for those with only AIIRBs (HR $=0.71,95 \% \mathrm{CI}=0.54-0.93)$. Regarding diabetes treatment, sulfonylurea and insulin were reported to cause hypoglycemia, which may explain the increased risk of hypoglycemia in the patients evaluated in the current study. However, as shown in Table 4, combining tramadol with metformin resulted in increased hypoglycemia ( $\mathrm{HR}=2.26,95 \% \mathrm{CI}=1.40-3.62)$. Guidelines for diabetes treatment from different countries frequently state that metformin does not increase hypoglycemia. Metformin activates adenosine monophosphate-activated protein kinase in the liver and muscles to improve glucose and lipid metabolism in diabetic humans; thus, metformin increases hepatic insulin sensitivity [16]. Another study revealed that tramadol enhances hepatic insulin sensitivity [17]. One possible explanation is that combining tramadol with metformin increased hepatic insulin sensitivity, resulting in hypoglycemia in diabetes. We suggest reducing the dose of metformin to avoid a hypoglycemic attack if the combination therapy with tramadol is necessary in diabetic Asian. Regarding the loop diuretics conducted in this study to increase hypoglycemia, only one table shows established and putative drugs that cause hypoglycemia from Williams Textbook of Endocrinology [18]. Diuretics was one of the putative drugs in this textbook. The Captopril Prevention Project suggested that conventional treatment with $\beta$-blocker and diuretics resulted in new-onset diabetes [19], which is an indirect effect for explaining our finding. Another finding in the current study is that tramadol plus AIIRBs resulted in less hypoglycemia. Several studies conducted on AIIRB have shown that these types of medication, including valsartan, losartan and candesartan, reduce new-onset diabetes. One study indicated the possible mechanism in which AIIRBs attenuate renin-angiotensin system activities induced by hyperglycemia or dyslipidemia. The net result in the islet cells was to preserve islet cells and avoid hyperglycemia instead of hypoglycemia [20].

Tramadol is prescribed as the active synthetic opioid analgesic drug in central organ and it can cause hypoglycemia after a blunted autonomic counter-regulatory reaction to diabetic patients. Tramadol can lower plasma glucose in diabetic rats because of an activation of mu opioid receptors (MOR) and this kind of activation results in a reduction of hepatic gluconeogenesis and also an increase of glucose utilization in peripheral tissue [21]. In the other study, tramadol has the effects on the central nervous system to interfere hepatic glucose utilization and it may cause the possibility of hypoglycemia [17]. Tramadol related to hypoglycemia may be based on the diabetic type such as beta cell failure or insulin resistance or the diabetic hormonal regulatory mechanism [22]. 


\section{Strengths and Limitations}

The strengths of our study are its population-based design, generalizability of findings and use of population-based data and the National Health Insurance Research Database (NHIRD) records with a very large sample size including study and control cohorts. In addition, NHIRD covers a highly representative sample of Taiwan's general population because the reimbursement policy is universal and operated by a single-buyer, the government in Taiwan. The accuracy of medical coding in the claims data may affect the data validity. All insurance claims should be scrutinized by medical reimbursement specialists and peer review. If these doctors or hospitals make wrong diagnoses or coding, they will be punished with a lot of penalties. Therefore, the diagnoses or coding in this study were highly reliable.

There are some study limitations. First, we could not collect other factors associated with hypoglycemic risk, such as ethanol use, or other lifestyle factors, such as diet and exercise pattern, from the NHIRD. Second, we could not record herbal or other traditional diabetes treatments, which may induce a risk of hypoglycemic attack. Third, bitter melon and okra are widely used throughout Asia as a staple food but it was also a limitation for our data form NHIRD. Fourth, poor compliance or adherence for medication use could be a factor for hypoglycemia but it was also difficult to use the National Health Insurance Research Database (HNIRD) data for analysis.

\section{Conclusions}

Tramadol use increases hypoglycemic risk in diabetic patients. In this study, we should pay more attention to prevent hypoglycemia attack in those high risk diabetic patients who were older, with chronic kidney disease and prescribed medications such as insulin, sulfonylurea, metformin and loop diuretics in Asia.

Author Contributions: Conception/Design: H.-H.C., C.-H.K., Provision of study material or patients: All authors, Collection and/or assembly of data: All authors, Data analysis and interpretation: All authors, Manuscript writing: All authors, Final approval of manuscript: All authors.

Acknowledgments: This work was supported by grants from the Ministry of Health and Welfare, Taiwan (MOHW107-TDU-B-212-123004), China Medical University Hospital (CMU106-ASIA-12, DMR-107-192); Academia Sinica Stroke Biosignature Project (BM10701010021); MOST Clinical Trial Consortium for Stroke (MOST 106-2321-B-039-005-); Tseng-Lien Lin Foundation, Taichung, Taiwan; and Katsuzo and Kiyo Aoshima Memorial Funds, Japan. The funders had no role in study design, data collection and analysis, decision to publish, or preparation of the manuscript. No additional external funding received for this study.

Conflicts of Interest: The authors declare no conflict of interest.

\section{References}

1. Gerstein, H.C.; Miller, M.E.; Byington, R.P.; Goff, D.C., Jr.; Bigger, J.T.; Buse, J.B.; Cushman, W.C.; Genuth, S.; Ismail, B.F.; Grimm, R.H., Jr.; et al. Effects of intensive glucose lowering in type 2 diabetes. N. Engl. J. Med. 2008, 358, 2545-2559. [PubMed]

2. Zoungas, S.; Patel, A.; Chalmers, J.; De Galan, B.E.; Li, Q.; Billot, L.; Woodward, M.; Ninomiya, T.; Neal, B.; MacMahon, S.; et al. Severe hypoglycemia and risks of vascular events and death. N. Engl. J. Med. 2010, 363, 1410-1418. [CrossRef] [PubMed]

3. Hsu, P.F.; Sung, S.H.; Cheng, H.M.; Yeh, J.S.; Liu, W.L.; Chan, W.L.; Chen, C.H.; Chou, P.; Chuang, S.Y. Association of clinical symptomatic hypoglycemia with cardiovascular events and total mortality in type 2 diabetes: A nationwide population-based study. Diabetes Care 2013, 36, 894-900. [CrossRef] [PubMed]

4. Whitmer, R.A.; Karter, A.J.; Yaffe, K.; Quesenberry, C.P., Jr.; Selby, J.V. Hypoglycemic episodes and risk of dementia in older patients with type 2 diabetes mellitus. JAMA 2009, 301, 1565-1572. [CrossRef] [PubMed]

5. Punthakee, Z.; Miller, M.E.; Launer, L.J.; Williamson, J.D.; Lazar, R.M.; Cukierman, Y.T.; Seaquist, E.R.; Ismail, B.F.; Sullivan, M.D.; Lovato, L.C.; et al. Poor cognitive function and risk of severe hypoglycemia in type 2 diabetes: Post hoc epidemiologic analysis of the ACCORD trial. Diabetes Care 2012, 35, 787-793. [CrossRef] [PubMed] 
6. Seaquist, E.R.; Anderson, J.; Childs, B.; Cryer, P.; Dagogo-Jack, S.; Fish, L.; Heller, S.R.; Rodriguez, H.; Rosenzweig, J.; Vigersky, R.; et al. Hypoglycemia and diabetes: A report of a workgroup of the American Diabetes Association and the Endocrine Society. Diabetes Care 2013, 36, 1384-1395. [CrossRef] [PubMed]

7. Wang, C.Y.; Lin, C.L.; Huang, T.S.; Chien, M.N.; Hsieh, S.H.; Huang, Y.Y.; Shih, K.C.; Tu, S.T.; Chang, C.T.; Chien, N.H.; et al. Inertia on hypoglycemia: Highlight from a Taiwan subgroup analysis of Real-Life Effectiveness and Care Patterns of Diabetes Management (RECAP-DM) study. Diabetes Res. Clin. Pract. 2012, 98, 61-67. [CrossRef] [PubMed]

8. American Diabetes Association. (9) Microvascular complications and foot care. Diabetes Care 2015, 38, 58-66. [CrossRef] [PubMed]

9. Fournier, J.P.; Azoulay, L.; Yin, H.; Montastruc, J.L.; Suissa, S. Tramadol use and the risk of hospitalization for hypoglycemia in patients with non-cancer pain. JAMA Intern. Med. 2015, 175, 186-193. [CrossRef] [PubMed]

10. Budnitz, D.S.; Lovegrove, M.C.; Shehab, N.; Richards, C.L. Emergency hospitalizations for adverse drug events in older Americans. N. Engl. J. Med. 2011, 365, 2002-2012. [CrossRef] [PubMed]

11. Insurance, BoNH. The National Health Insurance Annual Statistical Report, 2010; Bureau of National Health Insurance: Taipei, Taiwan, 2010.

12. Bourne, C.; Gouraud, A.; Daveluy, A.; Grandvuillemin, A.; Auriche, P.; Descotes, J.; Vial, T.; French Association of Regional Pharmacovigilance Centres. Tramadol and hypoglycaemia: Comparison with other step 2 analgesic drugs. Br. J. Clin. Pharmacol. 2013, 75, 1063-1067. [CrossRef] [PubMed]

13. Yamada, J.; Sugimoto, Y.; Kimura, I.; Takeuchi, N.; Horisaka, K. Serotonin-induced hypoglycemia and increased serum insulin levels in mice. Life Sci. 1989, 45, 1931-1936. [CrossRef]

14. Chi, T.C.; Ho, Y.J.; Chen, W.P.; Chi, T.L.; Lee, S.S.; Cheng, J.T.; Su, M.J. Serotonin enhances beta-endorphin secretion to lower plasma glucose in streptozotocin-induced diabetic rats. Life Sci. 2007, 80, 1832-1838. [CrossRef] [PubMed]

15. Liu, I.M.; Niu, C.S.; Chi, T.C.; Kuo, D.H.; Cheng, J.T. Investigations of the mechanism of the reduction of plasma glucose by cold-stress in streptozotocin-induced diabetic rats. Neuroscience 1999, 92, 1137-1142. [CrossRef]

16. Cheng, A.Y.; Fantus, I.G. Oral antihyperglycemic therapy for type 2 diabetes mellitus. CMAJ 2005, 172, 213-226. [CrossRef] [PubMed]

17. Choi, S.B.; Jang, J.S.; Park, S. Tramadol enhances hepatic insulin sensitivity via enhancing insulin signaling cascade in the cerebral cortex and hypothalamus of $90 \%$ pancreatectomized rats. Brain Res. Bull. 2005, 67, 77-86. [CrossRef] [PubMed]

18. Kronenberg, H.M. Glucose homeostasis and hypoglycemia. In Williams Textbook of Endocrinology, 11th ed.; Melmed, S., Polonsky, K.S., Eds.; Elsevier: Amsterdam, The Netherlands, 2007; Volume 33, pp. 1503-1533.

19. Hansson, L.; Lindholm, L.H.; Niskanen, L.; Lanke, J.; Hedner, T.; Niklason, A.; Luomanmäki, K.; Dahlöf, B.; De Faire, U.; Mörlin, C.; et al. Effect of angiotensin-converting-enzyme inhibition compared with conventional therapy on cardiovascular morbidity and mortality in hypertension: The Captopril Prevention Project (CAPPP) randomised trial. Lancet 1999, 353, 611-616. [CrossRef]

20. Leung, P.S.; Carlsson, P.O. Pancreatic islet renin angiotensin system: Its novel roles in islet function and in diabetes mellitus. Pancreas 2005, 30, 293-298. [CrossRef] [PubMed]

21. Cheng, J.T.; Liu, I.M.; Chi, T.C.; Tzeng, T.F.; Lu, F.H.; Chang, C.J. Plasma-glucose-lowering effect of tramadol in streptozotocin-induced diabetic rats. Diabetes 2001, 50, 2815-2821. [CrossRef] [PubMed]

22. Briscoe, V.J.; Davis, S.N. Hypoglycemia in type 1 and type 2 diabetes: Physiology, pathophysiology and management. Clin. Diabetes 2006, 24, 115-124. [CrossRef]

(C) 2018 by the authors. Licensee MDPI, Basel, Switzerland. This article is an open access article distributed under the terms and conditions of the Creative Commons Attribution (CC BY) license (http://creativecommons.org/licenses/by/4.0/). 